\title{
Comparative ophthalmic assessment of patients receiving tafenoquine or chloroquine/primaquine in a randomized clinical trial for Plasmodium vivax malaria radical cure
}

\author{
Sukhuma Warrasak • Ataya Euswas · Mark M. Fukuda • Mali Ittiverakul • \\ R. Scott Miller · Srivicha Krudsood $\cdot$ Colin Ohrt
}

Received: 16 April 2018/Accepted: 11 August 2018/Published online: 29 September 2018

(C) The Author(s) 2018

\begin{abstract}
Purpose Ophthalmic safety observations are reported from a clinical trial comparing tafenoquine (TQ) efficacy and safety versus sequential chloroquine (CQ)/primaquine (PQ) for acute Plasmodium vivax malaria.

Methods In an active-control, double-blind study, 70 adult subjects with microscopically confirmed $P$. vivax malaria were randomized $(2: 1)$ to receive $400 \mathrm{mg}$ $\mathrm{TQ} \times 3$ days or $1500 \mathrm{mg} \mathrm{CQ} \times 3$ days then $15 \mathrm{mg}$

Disclaimer This manuscript was reviewed by the Walter Reed Army Institute for Research and the United States Army Medical Research and Material Command. There is no objection to its publication or dissemination. The opinions reflected herein reflect those of the authors and do not necessarily reflect the official policy, position or opinions of the US Army, the US Department of Defense, or the US Government.
\end{abstract}

\section{S. Warrasak $(\bowtie) \cdot$ A. Euswas}

Department of Ophthalmology, Faculty of Medicine,

Ramathibodi Hospital, Mahidol University,

Bangkok 10400, Thailand

e-mail: sukhuma@csloxinfo.com

A. Euswas

e-mail: ataya@csloxinfo.com

Present Address:

S. Warrasak

The Eye Center, Debaratana Medical Center, Ramathibodi Hospital Faculty, Mahidol University, 270 Rama 6 Road, Rajthevi, Phyathai, Bangkok 10400, Thailand
PQ $\times 14$ days. Main outcome measures: clinically relevant changes at Day 28 and Day 90 versus baseline in the ocular examination, color vision evaluation, and corneal and retinal digital photography.

Results Post-baseline keratopathy occurred in 14/44 $(31.8 \%)$ patients with TQ and $0 / 24$ with CQ/PQ $(P=0.002)$. Mild post-baseline retinal findings were reported in 10/44 $(22.7 \%)$ patients receiving TQ and $2 / 24(8.3 \%)$ receiving CQ/PQ $(P=0.15$; treatment difference $14.4 \%, 95 \%$ CI $-5.7,30.8)$. Masked evaluation of retinal photographs identified a retinal hemorrhage in one TQ patient (Day 90) and a slight increase in atrophy from baseline in one TQ and one $\mathrm{CQ} / \mathrm{PQ}$ patient. Visual field sensitivity (Humphrey ${ }^{\mathrm{TM}}$ 10-2 test) was decreased in 7/44 (15.9\%) patients receiving TQ and 3/24 (12.5\%) receiving CQ/PQ; all

Present Address:

A. Euswas

The Bangkok Eye Center, Bangkok Hospital Medical Group, Bangkok 10310, Thailand

M. M. Fukuda · M. Ittiverakul · R. S. Miller Department of Immunology and Medicine, Armed Forces Research Institute of Medical Sciences (AFRIMS), 315/6 Rajvithi Road, Bangkok 10400, Thailand

e-mail: mark.fukuda@afrims.org

M. Ittiverakul

e-mail: mali.ittiverakul@afrims.org

R. S. Miller

e-mail: scott.miller@gatesfoundation.org 
cases were $<5 \mathrm{~dB}$. There were no clinically relevant changes in visual acuity or macular function tests.

Conclusions There was no evidence of clinically relevant ocular toxicity with either treatment. Mild keratopathy was observed with TQ, without conclusive evidence of early retinal changes. Eye safety monitoring continues in therapeutic studies of lowdose tafenoquine (300 $\mathrm{mg}$ single dose).

Clinical trial registration Clinicaltrials.gov identifier: NCT01290601.

Keywords Ophthalmic safety - Tafenoquine ·

Chloroquine $\cdot$ Primaquine $\cdot$ Keratopathy

Retinopathy $\cdot$ Plasmodium vivax . Clinical trial .

Malaria

$\begin{array}{ll}\text { Abbreviations } \\ \text { CQ } & \text { Chloroquine } \\ \text { HCQ } & \text { Hydroxychloroquine } \\ \text { PQ } & \text { Primaquine } \\ \text { TQ } & \text { Tafenoquine }\end{array}$

\section{Background}

Plasmodium vivax malaria is endemic in Central and South America, South East Asia, Oceania, and parts of

\section{Present Address:}

R. S. Miller

The Malaria Program, Bill and Melinda Gates Foundation, PO Box 23350, Seattle, WA 98102, USA

S. Krudsood

Faculty of Tropical Medicine, Mahidol University, 420/6

Ratchawithi Road, Ratchathewi, Bangkok 10400,

Thailand

e-mail: srivicha.kru@mahidol.ac.th

C. Ohrt $(\square)$

Division of Experimental Therapeutics, Walter Reed Army Institute of Research, Silver Spring, USA

e-mail: colin@consortiumha.org

Present Address:

C. Ohrt

Consortium for Health Action, Savage, MN, USA

Present Address:

C. Ohrt

Phnom Penh, Cambodia

C. Ohrt

Hanoi, Vietnam
Africa. It represents a substantial health and economic burden with an estimated 132-391 million infections annually $[1,2]$. Despite its high prevalence, $P$. vivax malaria is considered a neglected disease and only recently has its true impact on morbidity and particularly mortality been acknowledged [2-4]. P. vivax treatment and control are complicated by the ability of the parasite to form hypnozoites. These develop from sporozoites that enter into a dormant state, rather than actively dividing in the host liver. Hypnozoites periodically reenter the development cycle causing clinical relapse weeks or months after the infectious inoculation.

Primaquine (PQ) remains the only agent available for hypnozoite eradication, termed 'radical cure'. PQ is given for 14 days following standard treatment with a blood schizonticide, usually chloroquine (CQ). However, patients often feel well after the first few days of commencing treatment and so adherence with 14-day PQ is problematic, and clinical effectiveness is therefore compromised [5]. Thus, there is a need to develop new treatments with improved convenience for patients that are safe and effective for $P$. vivax radical cure. In addition to relieving the clinical burden of repeated $P$. vivax relapses, the availability of an effective therapy for $P$. vivax radical cure has particular relevance for malaria elimination operations [6-9].

Tafenoquine (TQ) is an 8-aminoquinoline discovered and developed by the Walter Reed Army Institute of Research [10]. Chemically related to PQ, TQ has potent antihypnozoite activity [11], but has a 2-3week half-life [8, 12]. This raises the possibility of shorter treatment regimens for $P$. vivax radical cure.

This clinical trial was conducted under an NIH Challenge Grant entitled 'Tafenoquine, a Novel Drug for Malaria Prevention and Control.' The primary objective of this study was to determine if 3-day TQ monotherapy could clear and cure $P$. vivax blood-stage infection, with secondary objectives of rapidity of parasite clearance from the bloodstream, antihypnozoite activity, population pharmacokinetics, and safety. The comparator arm was standard sequential therapy with CQ followed by PQ. The efficacy and main safety results of this study have been previously reported [13]. Briefly, although there was no difference in efficacy in this study, the slow rate of parasite, gametocyte and fever clearance indicated that tafenoquine should not be used as monotherapy for radical cure of $P$. vivax malaria, at which point clinical development in this indication was paused [13]. 
More recently, GlaxoSmithKline and the Medicines for Malaria Venture (MMV) have been developing single-dose TQ plus CQ (TQ/CQ) for $P$. vivax radical cure. A dose-ranging clinical trial from this programme showed that a single $300 \mathrm{mg}$ TQ dose plus 3-day CQ prevented $P$. vivax relapse in $89 \%$ (95\% CI 77-95) of patients over a 6-month period compared with $37.5 \%$ (95\% CI 23-52) with CQ alone (treatment difference $51.7 \%$ [95\% CI 35-69], $P<0.0001$ ) [14]. In the same study, CQ plus $15 \mathrm{mg} \times 14$ days PQ prevented relapse in $77.3 \%$ (95\% CI 63-87) of patients [14].

CQ is an antimalarial drug with well-known ophthalmic toxicity [15]. Similar to several other drugs, such as amiodarone and tamoxifen [16, 17], CQ causes keratopathy (lipid deposits) in the cornea resulting in changes ranging from epithelial haze to a dense 'whorl' [17]. These findings are usually considered benign and reversible and, in the case of CQ, occur generally during extended use in rheumatic diseases [17]. However, retinal toxicity associated with CQ can lead to permanent vision impairment, which is dose and duration related $[18,19]$. Early CQ retinal toxicity can appear as mild retinal pigment epithelium changes [18].

A study published in 2010 found possible eye safety findings with TQ in a Phase III prophylaxis trial in Australian soldiers [20], though there were no baseline ocular data to evaluate a drug effect. Also, ocular safety evaluation was based on macular function testing plus digital photography without retinal examinations, so correlation between morphological changes and functional impairment was not possible.

In the ongoing $P$. vivax radical cure clinical trial program, TQ/CQ are co-administered, and it will not be possible to differentiate between CQ and TQ ocular safety findings. Here we report ophthalmic safety data from a randomized clinical trial of tafenoquine monotherapy in $P$. vivax radical cure. This study was designed to determine whether the previous eye findings with TQ had clinical significance and assess whether there is an ocular safety risk with TQ [13]. Thus, this study reports the only available data evaluating the ocular safety of TQ alone in $P$. vivax malaria patients.

\section{Methods}

Patients

Eligible subjects were 20-60 years old with microscopically confirmed $P$. vivax malaria (parasite density $>500$ and $<200,000 \mu \mathrm{L}^{-1}$ ). Exclusion criteria were: mixed malaria infection; severe vomiting; demonstrated glucose-6-phosphate dehydrogenase deficiency; history of antimalarial therapy within 30 days; clinically significant illness or abnormal laboratory values; history of allergy to study drugs or 8-aminoquinolines; use of an investigational drug within 30 days or 5 half-lives (whichever longer); history of previous eye surgery; corneal or retinal abnormalities; concomitant medication that would affect safety evaluations; and a clinical assessment of risk for acute angle closure glaucoma. A negative pregnancy test was required from all women of childbearing potential. Pre-menarchal, pregnant, and breastfeeding women were excluded.

\section{Design and procedures}

This randomized, active-control, double-blind, double-dummy study was conducted at the Bangkok Hospital for Tropical Diseases, Bangkok, Thailand, between September 2003 and January 2005. All drug treatments were supplied as capsules containing (free base equivalent): TQ $200 \mathrm{mg}$ (GlaxoSmithKline, UK), CQ 150 mg (AstraZeneca UK Ltd, UK), or PQ $15 \mathrm{mg}$ (Boucher and Muir Pty Ltd, Australia). Matching placebos were used to maintain study blinding.

The computer-generated randomization schedule was provided by GlaxoSmithKline. Eligible subjects were allocated a sequential randomization number and treatment allocated through the GlaxoSmithKline Registration and Medication Ordering System (RAMOS) in a 2:1 ratio to TQ or the comparator arm using blocks of six. Subjects, investigators and study staff remained blinded to treatment allocation throughout the study. Emergency unblinding was possible through RAMOS. There was one code break at Day 4 for a patient found to have a history of eye surgery/abnormality, and this patient was withdrawn from the study.

The study design is shown in Fig. 1. Patients were randomized to receive either TQ $400 \mathrm{mg}$ for 3 days (Day 0,1 and 2) or CQ $600 \mathrm{mg}$ for 2 days (Day 0 and 1) followed by CQ $300 \mathrm{mg}$ for 1 day (Day 2) then PQ $15 \mathrm{mg}$ for 14 days (Days 3-16). All doses are given as free base equivalents. Follow-up continued to Day 120. All doses were directly observed and given with food. Vomiting within $1 \mathrm{~h}$ of dosing was to result in redosing. Vomiting of any subsequent dose was to lead 
Fig. 1 Study design and patient numbers

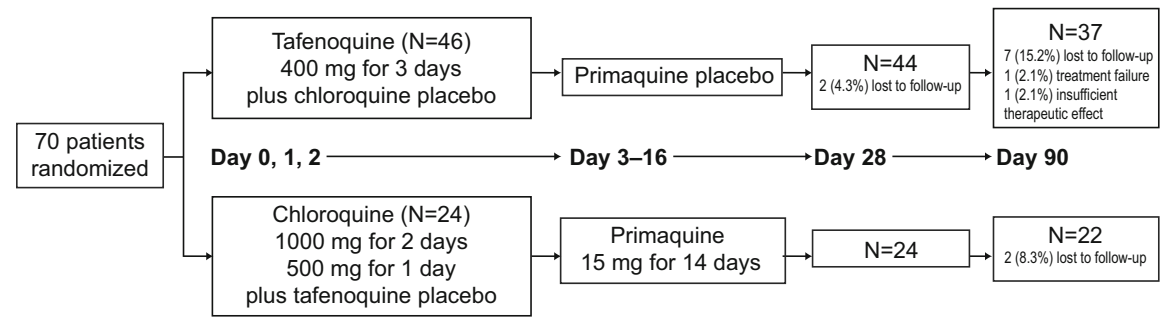

to patient withdrawal from the study and administration of rescue medication. Patients were hospitalized for 29 days of observation, and requested to remain in a malaria-free region for 60 days thereafter.

\section{Ophthalmic assessments}

All ophthalmic assessments were performed with patients and investigators blinded to study treatment allocation. Ophthalmic assessments were conducted at baseline (within $36 \mathrm{~h}$ of receiving the first dose of study drug), Day 28 and Day 90 at the Ramathibodi Faculty Hospital, Bangkok, Thailand. Clinically relevant changes versus baseline were recorded as adverse events using the MedDRA coding system (version 8.1). Patients with clinically relevant changes were followed up until resolution or following improvement to a degree that clinical monitoring was no longer required. Ophthalmic assessment criteria for patient study withdrawal were prospectively defined.

Best spectacle-corrected visual acuity was assessed with the high contrast visual acuity (HCVA) test using the Early Treatment Diabetic Retinopathy Study (ETDRS) chart with letters. A clinically relevant change was defined as a decrease in visual acuity of $0.08 \log$ MAR (minimum angle of resolution), equivalent to five letters decrease on the high contrast chart or one line. Patients with decreased vision of 0.3 $\log$ MAR, equivalent to 15 letters decrease on the high contrast chart or three lines, were to be withdrawn.

Ocular examination consisted of slit-lamp biomicroscopy of the anterior segment, applanation tonometry, and dilated fundus examination of the central and peripheral fundus. Ocular images (corneal and retinal digital photography) were documented. Clinically relevant findings in the cornea were graded as Grade $0=$ no haze, Grade $1=$ trace to minimal haze, Grade 2 = moderate haze, Grade 3 = marked haze, Grade 4 = significant haze. Digital photographs were taken of the cornea with the Haag-Streit EyeCap ${ }^{\mathrm{TM}}$ system (Haag-Streit AG, Switzerland) which included a slitlamp biomicroscope BQ900 (magnification 16×) and a computer loaded with the software. Retinal changes in the macula were assessed and collected as (in increasing intensity): slight granularity, mottling of pigmentation, definite area of depigmentation and/or hyperpigmentation, and ring of depigmentation surrounded by a ring of hyperpigmentation (any degree of bull's eye maculopathy). Any patient with any degree of bull's eye retinopathy, confirmed by clinical assessment, digital photography, or fundus fluorescein angiography, was to be withdrawn from the study. Digital images of the retina were captured with the fundus camera of the Topcon IMAGEnet ${ }^{\mathrm{TM}} 2000$ system (TRC-50IX, IMAGEnet ${ }^{\mathrm{TM}}$ 2000, Topcon, Japan) at $20^{\circ}, 35^{\circ}$, and $50^{\circ}$ during the study at baseline, Day 28 and Day 90. A masked review of the photographs was conducted at the Fundus Photograph Reading Center, University of Wisconsin.

Macular function tests were: Amsler grid test, a Humphrey ${ }^{\mathrm{TM}}$ 10-2 visual field test, the macular stress test and color vision evaluation using the pseudoisochromatic plate (PIP) ishihara compatible test, and the L'Anthony 40 hue test. For the Amsler grid test, the presence of a repeatable (two assessments within $10 \mathrm{~min}$ ) area of distortion (metamorphopsia) or scotoma covering more than one grid block $\left(>1^{\circ}\right.$ of visual angle) was to result in patient withdrawal. To identify macular disease, a Humphrey ${ }^{\mathrm{TM}}$ 10-2 visual field test was performed. Presence of a treatmentemergent scotoma in two tests at least 30 min apart with a $5 \mathrm{~dB}$ decrease in sensitivity (local or overall visual field) was considered clinically relevant [21], and a decrease in sensitivity of at least $10 \mathrm{~dB}$ was to lead to patient withdrawal. For the macular stress test, using the time to read the line above the best spectaclecorrected visual acuity as an endpoint, an increase of $30 \mathrm{~s}$ in recovery time versus baseline was considered clinically relevant. For assessment of color vision, an 
increase of two in the number of PIP plates missed required further investigation using the L'Anthony 40 hue test; greater than two reversals was clinically significant. Subjects with four or more reversals on the L'Anthony hue 40 test were to be withdrawn.

\section{Statistical analysis}

The evaluation of all ophthalmic safety data was made in the safety population, including all subjects that received at least one dose of study medication, based on actual available observations at each time point. As this study was designed for an efficacy endpoint, no formal sample size calculation was made for the ophthalmic safety outcomes. Confidence intervals (CI) and $P$ values for the differences in binomial proportions are exact and have been produced by the inversion of two onesided tests using the standardized (score) statistic. Calculations were performed using the StatXact statistical software (version 6.3). $P$ values are presented where outcomes were significantly different. As this study was not powered for assessment of ophthalmic outcomes, non-significant $P$ values are presented with 95\% confidence intervals for the treatment difference to provide some indication of trend.

\section{Results}

Patient baseline characteristics are summarized in Table 1; they were similar for the two treatment groups. All patients were from Myanmar or Thailand. Figure 1 shows the study design and patient numbers included for the ophthalmic assessments. The intention-to-treat population included 70 patients, 46 randomized to $\mathrm{TQ}$ and 24 to $\mathrm{CQ} / \mathrm{PQ}$. All patients were fully compliant with medication. At Day 28, 44 patients in the TQ arm and 24 in the CQ/PQ arm were available for analysis, whereas by Day 90, 7/46 $(15.2 \%)$ in the TQ arm versus $2 / 24(8.3 \%)$ in the $\mathrm{CQ} / \mathrm{PQ}$ arm were lost to follow-up.

Adverse events recorded within the term 'eye disorders' were more common with TQ (17/46 [37.0\%]) versus CQ/PQ (4/24 [16.7\%]). In the TQ group $(n=46)$ adverse events were described as keratopathy $(n=14,30.4 \%)$, retinopathy/retinal disorder $(n=10,21.7 \%)$, and eye irritation $(n=1$, $2.2 \%)$. In the CQ/PQ group $(n=24)$, there were two cases of conjunctivitis $(8.3 \%)$ and one each of retinopathy, eye inflammation and eye pain (4.2\% each).

\section{Corneal assessments}

The outcomes of ophthalmic clinical assessments are summarized in Table 2. At baseline, 1/46 (2.2\%) patients in the TQ arm and 0/24 in the CQ/PQ arm had keratopathy. At any follow-up visits, (Day 28 or Day 90), keratopathy was observed in 14/44 (31.8\%) patients with TQ versus none with $\mathrm{CQ} / \mathrm{PQ}$ $(P=0.002)$. At Day 28, $12 / 44(27.3 \%)$ patients receiving TQ had keratopathy; $11 / 44$ (25.0\%) were

Table 1 Summary of patient baseline characteristics

\begin{tabular}{lll}
\hline Characteristic & Tafenoquine $(N=46)$ & Chloroquine/primaquine $(N=24)$ \\
\hline Median age, years (range) & $23.5(20-43)$ & $30.0(20-55)$ \\
Male sex, $n(\%)$ & $37(80.4)$ & $20(83.3)$ \\
$P$. vivax total parasites count, median parasites $\mu \mathrm{L}^{-1}($ range) & $4000(200-44,000)$ & $2730(600-30,000)$ \\
$P$. vivax gametocytes count, median parasites $\mu \mathrm{L}^{-1}($ range $)$ & $n=26$ & $n=15$ \\
& $80(20-640)$ & $60(40-280)$ \\
Mean temperature, ${ }^{\circ} \mathrm{C}(\mathrm{SD})$ (range) & $37.0(0.9)[36.0-40.0]$ & $36.8(0.7)[36.0-39.3]$ \\
Previous malaria (yes), $n(\%)$ & $30(65.2)$ & $16(66.7)$ \\
Malaria episodes in previous 6 months, $n(\%)$ & & $10(62.5)$ \\
0 & $14(46.7)$ & $4(25.0)$ \\
1 & $11(36.7)$ & $2(12.5)$ \\
2 & $3(10.0)$ & 0 \\
$\geq 3$ & $2(6.7)$ & $0.6(0.2-6.3)$ \\
Median time since last malaria, years (range) & $0.3(0.2-1.8)$ & \\
\hline
\end{tabular}


Table 2 Ocular findings by clinical assessment in P. vivax malaria patients treated with tafenoquine monotherapy or chloroquine/ primaquine sequential therapy

\begin{tabular}{|c|c|c|c|c|}
\hline Ocular findings & $\begin{array}{l}\text { Baseline } \\
(N=46)\end{array}$ & $\begin{array}{l}\text { Day } 28 \\
(N=44)\end{array}$ & $\begin{array}{l}\text { Day } 90 \\
(N=37)\end{array}$ & $\begin{array}{l}\text { Post-baseline } \\
\text { total }(N=44)\end{array}$ \\
\hline \multicolumn{5}{|l|}{ Tafenoquine } \\
\hline Keratopathy (direct examination), patients & $1(2.2)$ & $12(27.3)^{\mathrm{a}}$ & $7(18.9)$ & $14(31.8)$ \\
\hline Left eye & 0 & $12(27.3)^{\mathrm{a}}$ & $7(18.9)$ & $14(31.8)$ \\
\hline Right eye & $1(2.2)$ & $12(27.3)^{\mathrm{a}}$ & $6(16.2)$ & $14(31.8)$ \\
\hline Retinal findings (direct examination), patients & $1(2.2)$ & $9(20.5)$ & $8(21.6)$ & $10(22.7)$ \\
\hline Left eye & $1(2.2)$ & $9(20.5)$ & $8(21.6)$ & $10(22.7)$ \\
\hline Right eye & $1(2.2)$ & $8(18.2)$ & $7(18.9)$ & $9(20.5)$ \\
\hline Keratopathy and retinal findings ${ }^{b}$ & 0 & $6(13.6)$ & $3(8.1)$ & $7(15.9)$ \\
\hline Decreased sensitivity on visual field testing, patients ${ }^{\mathrm{c}}$ & $1(2.2)$ & $4(9.1)$ & $4(10.8)$ & $7(15.9)$ \\
\hline Left eye & 0 & $2(4.5)$ & $4(10.8)$ & $5(11.4)$ \\
\hline Right eye & $1(2.2)$ & $4(9.1)$ & $1(2.7)$ & $5(11.4)$ \\
\hline Retinal findings plus decreased sensitivity, patients & 0 & $1(2.3)$ & $1(2.7)$ & $2(4.5)^{\mathrm{d}}$ \\
\hline Left eye & 0 & $1(2.3)$ & $1(2.7)$ & $2(4.5)^{\mathrm{d}}$ \\
\hline Right eye & 0 & $1(2.3)$ & $1(2.7)$ & $2(4.5)^{\mathrm{d}}$ \\
\hline Chloroquine/primaquine & $(N=24)$ & $(N=24)$ & $(N=22)$ & $(N=24)$ \\
\hline Keratopathy (direct examination) & 0 & 0 & 0 & 0 \\
\hline Left eye & 0 & 0 & 0 & 0 \\
\hline Right eye & 0 & 0 & 0 & 0 \\
\hline Retinal findings (direct examination), patients & $2(8.3)^{\mathrm{e}}$ & $2(8.3)^{\mathrm{e}}$ & $1(4.5)$ & $2(8.3)^{\mathrm{e}}$ \\
\hline Left eye & $1(4.2)^{\mathrm{e}}$ & $2(8.3)^{\mathrm{e}}$ & $1(4.5)$ & $2(8.3)^{\mathrm{e}}$ \\
\hline Right eye & $2(8.3)^{\mathrm{e}}$ & $2(8.3)^{\mathrm{e}}$ & $1(4.5)$ & $2(8.3)^{\mathrm{e}}$ \\
\hline Decreased sensitivity on visual field testing, patients ${ }^{\mathrm{c}}$ & 0 & $3(12.5)^{\mathrm{e}}$ & 0 & $3(12.5)^{\mathrm{e}}$ \\
\hline Left eye & 0 & $3(12.5)^{\mathrm{e}}$ & 0 & $3(12.5)^{\mathrm{e}}$ \\
\hline Right eye & 0 & $2(8.3)^{\mathrm{e}}$ & 0 & $2(8.3)^{\mathrm{e}}$ \\
\hline Retinal findings plus decreased sensitivity, patients & 0 & $2(8.3)^{\mathrm{e}}$ & 0 & $2(8.3)^{\mathrm{e}}$ \\
\hline Left eye & 0 & $2(8.3)^{\mathrm{e}}$ & 0 & $2(8.3)^{\mathrm{e}}$ \\
\hline Right eye & 0 & $2(8.3)^{\mathrm{e}}$ & 0 & $2(8.3)^{\mathrm{e}}$ \\
\hline
\end{tabular}

Numbers are $n(\%)$

${ }^{\mathrm{a}} 11 / 44(25.0 \%)$ Grade I, $1 / 44(2.3 \%)$ Grade II

${ }^{\mathrm{b}}$ All patients had bilateral findings

${ }^{\mathrm{c}}$ Abnormal Humphrey ${ }^{\mathrm{TM}}$ 10-2 test; decreases in sensitivity were $<5 \mathrm{~dB}$ (not clinically relevant) in all cases

${ }^{\mathrm{d}}$ Including one patient who had decreased sensitivity in the left eye at Day 90 and retinal findings in the left eye on Day 28

e Including one patient with a dot hemorrhage near the fovea in both eyes at baseline and Day 28. Day 90 retinal examination was normal for both eyes. Decreased sensitivity on visual field testing was observed at Day 28 in both eyes and was normal at other assessments

Grade I, 1/44 (2.3\%) was Grade II. All of these patients had keratopathy in both eyes. By Day 90, keratopathy had resolved in 5/12 patients. Of the remaining seven patients, two were lost to follow-up at Day 90 and four had keratopathy reported as ongoing, plus one had a minor Grade I keratopathy. An additional two patients in the TQ arm developed Grade I new onset keratopathy by Day 90 . In all cases, the keratopathy was 

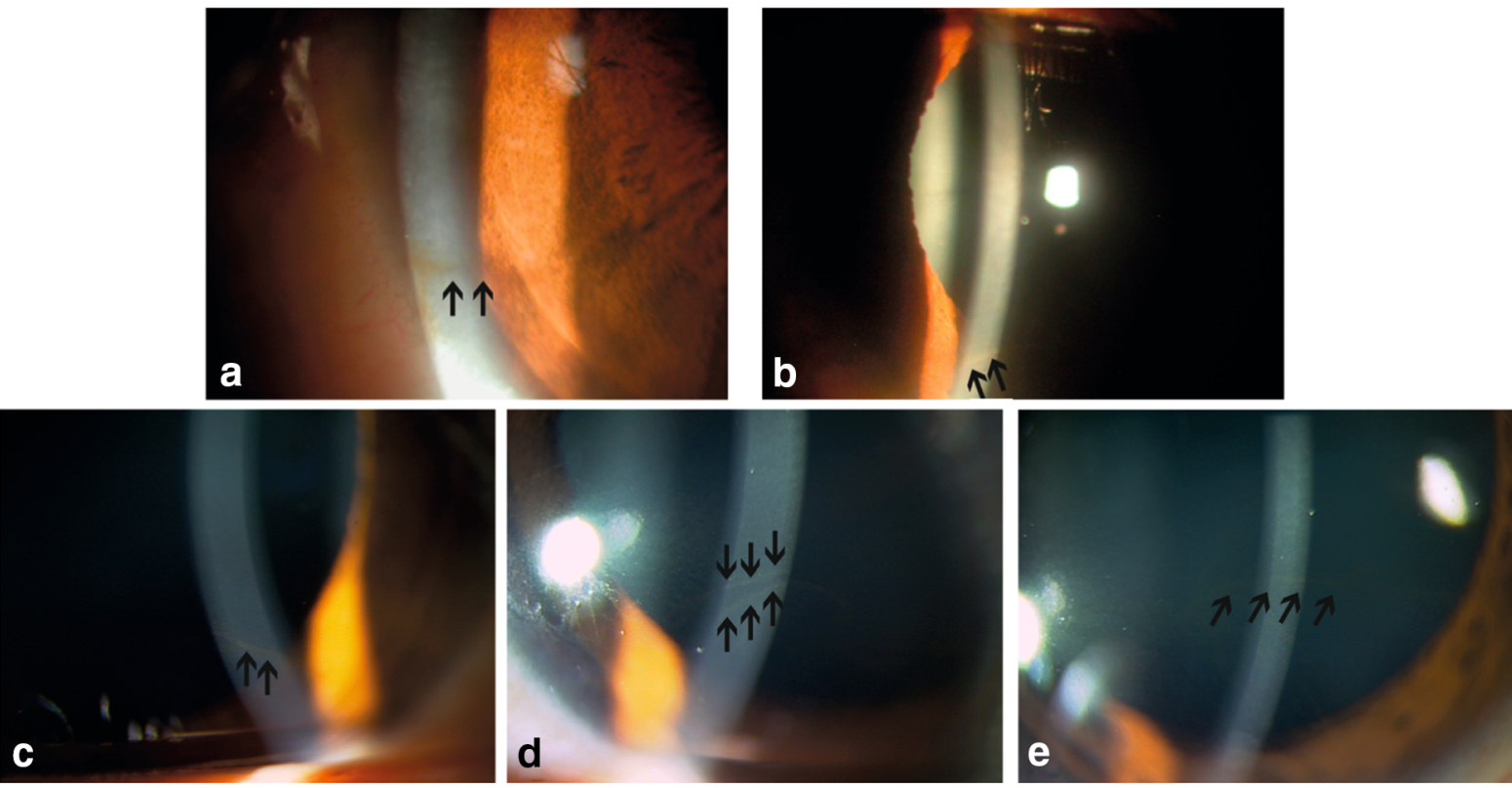

Fig. 2 Treatment-emergent keratopathy at Day 28 in four $P$. vivax malaria patients receiving tafenoquine; corneal changes were seen clinically as brown pigment deposits in the lower part

located in the inferior part of the cornea and did not involve the optical zone (Fig. 2). None of the patients who had keratopathy required further follow-up after Day 90 as keratopathy was mild and did not affect vision.

\section{Retinal assessments}

Baseline clinical fundus examination revealed one case of bilateral retinal dot hemorrhage in the CQ/PQ group, probably related to $P$. vivax malaria (Fig. 3a). In the TQ group, there was one case of post-baseline unilateral (in the left eye) retinal hemorrhage on Day 90 (Fig. 3b).

Retinal abnormalities were described as mottling of the retinal pigment epithelium, a group of yellow pigment deposits, or hypopigmentation (Fig. 4). Baseline retinal abnormalities were noted clinically in $1 / 46$ (2.2\%) patients in the TQ group and $2 / 24$ (8.3\%) in the $\mathrm{CQ} / \mathrm{PQ}$ group. Post-baseline retinal findings were reported on clinical examination in 10/44 (22.7\%) patients with TQ and $2 / 24(8.3 \%)$ in the CQ/PQ group $(P=0.15$, treatment difference $14.4 \% ; 95 \% \mathrm{CI}-5.7$, 30.8) (Table 2). At Day 28, 9/44 (20.5\%) patients receiving TQ had retinal findings reported (8/44 in both eyes, 1/44 in left eye only). At Day 90, one patient of the cornea (black arrows) and did not involve the optical zone. Images $\mathbf{c}$ and $\mathbf{d}$ are from the same individual

had been lost to follow-up, retinal findings had resolved in one patient and an additional patient had changes from baseline. Thus, 8/37 (21.6\%) of patients in the TQ group had retinal findings at Day 90 (7/37 in both eyes, 1/37 in left eye only).

In the CQ/PQ group, 1/24 (4.2\%) patients had retinal findings on clinical examination of mild pigmentation with abnormal assessments at Day 28 and 90 for the left eye and at Days 0, 28, and 90 for the right eye.

Clinical observations at Day 28 led to further investigation by fundus fluorescein angiogram (FFA) on one TQ patient with retinal findings (Fig. 4) and in one CQ/PQ patient with decreased sensitivity and retinal findings. Note that FFA was not pre-specified in the protocol, but conducted ad hoc only in these two patients in response to clinical findings. In the TQ patient, for whom retinal pigment epithelium alterations in the left eye were seen clinically as a group of yellow pigment deposits or hypopigmentation as shown in Fig. 4, abnormal FFA findings showed pinpoints of hyperfluorescence in the early phase of the angiograms and persisted through the late frames (Fig. 5). These hyperfluorescence spots corresponded to the focal depigmented retinal pigment epithelial cells that were observed fundoscopically (circled areas 
Fig. 3 a Baseline retinal hemorrhage (black arrow) at Day 0 in both eyes of a patient infected with $P$. vivax. b Post-baseline whitecentered retinal hemorrhage (black arrow) noted in the left eye of one patient in the tafenoquine group on Day 90 a

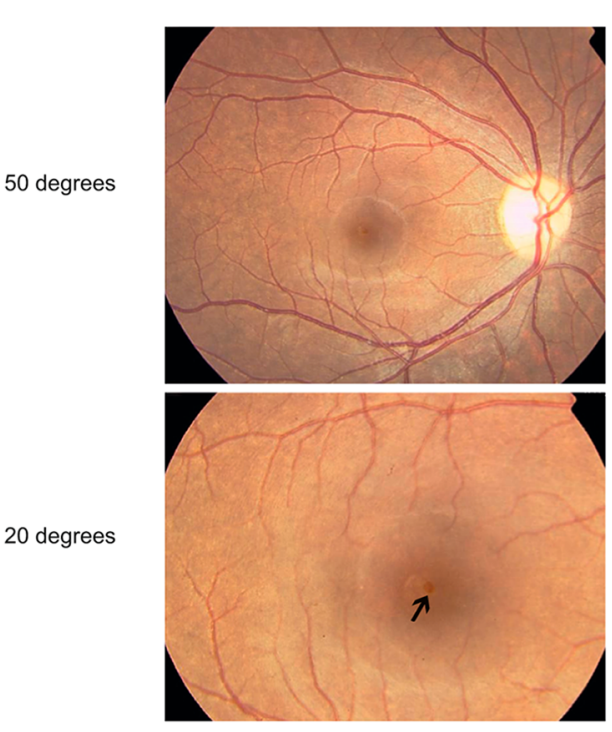

b
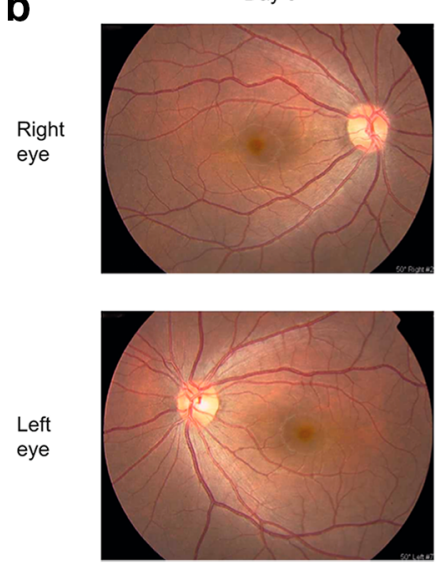

Left eye

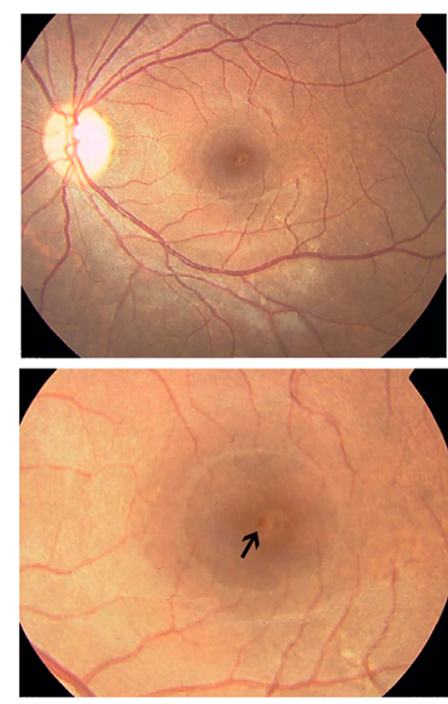

Day 90
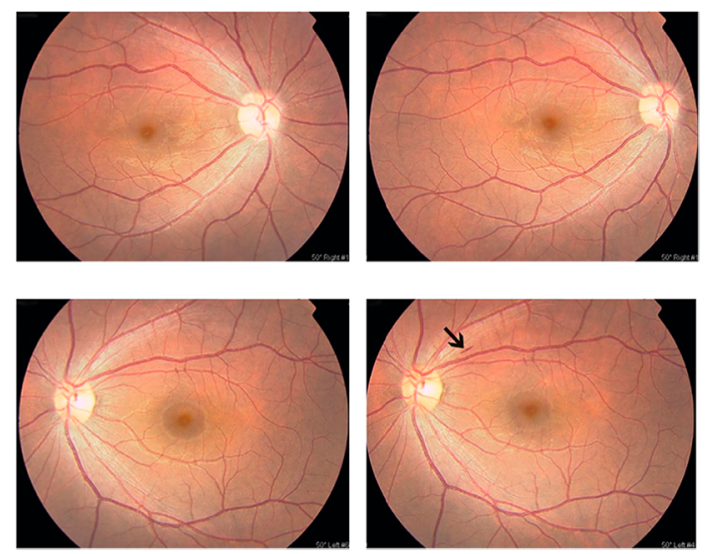

in Figs. 4, 5). However, as there was no baseline FFA, it is not clear whether these observations were related to drug treatment. No subsequent FFA assessments were obtained at Day 90 as retinal findings were stable, though subsequent review of digital photographs indicated a slight change in retinal pigment epithelium at Day 90. In this patient, threshold sensitivity in the left eye at Day 90 was decreased by $<5 \mathrm{~dB}$ and other macular function tests were unremarkable. For the patient receiving CQ/PQ who had retinal pigment epithelium changes, no FFA abnormalities were detected.

On masked digital photograph review by an independent reviewer, there were no changes from baseline noted at Day 28 for either group. At Day 90, apart from the patient with a retinal hemorrhage in the left eye, one TQ patient had a slight increase in atrophy versus baseline and one CQ/PQ patient had retinal atrophy at baseline and a slight increase in atrophy versus baseline at Day 90.

Visual acuity, macular function tests, and visual field testing

There were no clinically relevant changes in visual acuity or macular function tests (Table 3). At baseline, $1 / 46(2.2 \%)$ patients in the TQ group had an abnormal Humphrey ${ }^{\mathrm{TM}}$ 10-2 test in the right eye, which was not evident at later assessments. Post-baseline, a similar proportion of patients in the TQ group (7/44 [15.9\%]) 
Day 0

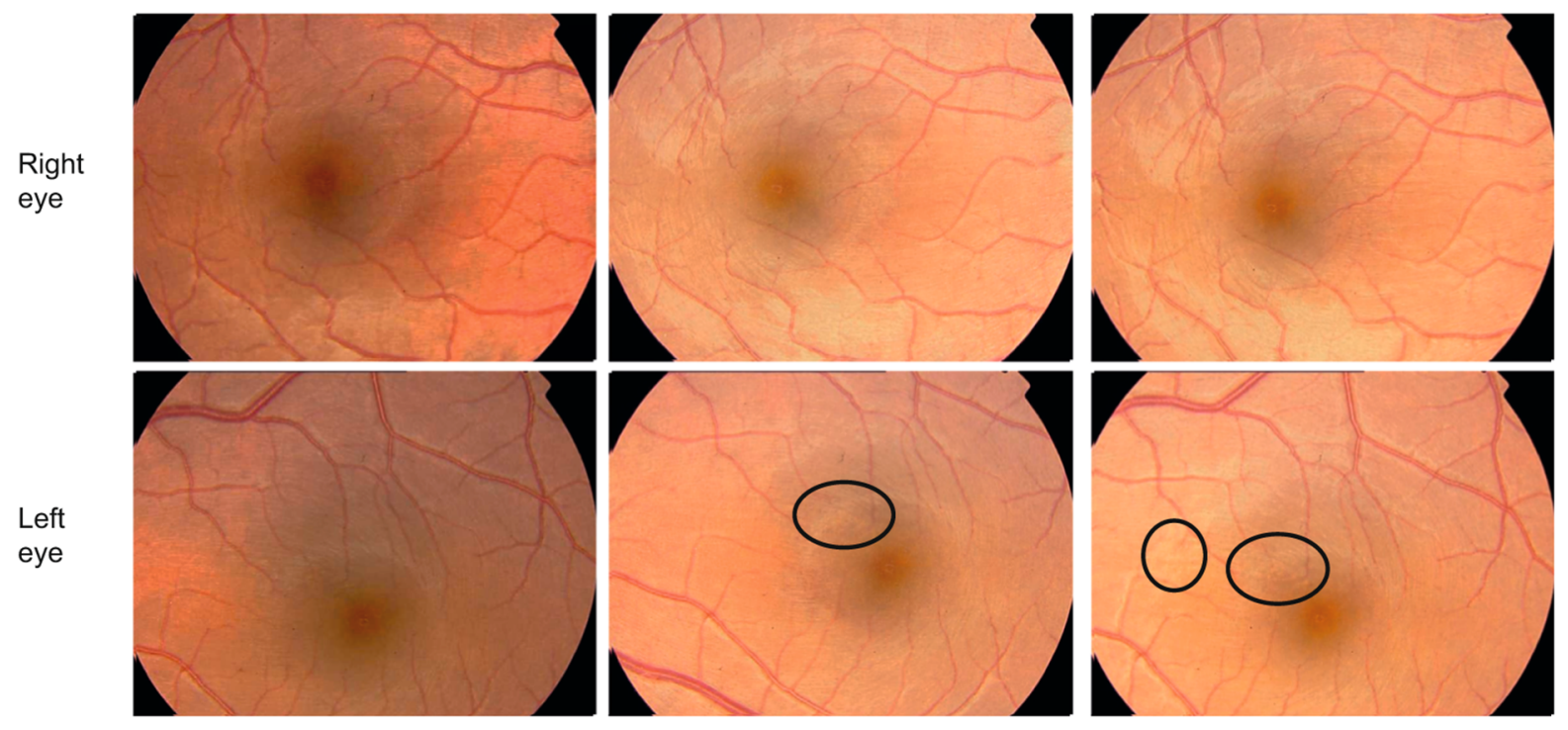

Fig. 4 In a $P$. vivax malaria patient receiving tafenoquine, retinal pigment epithelium alteration, seen as a small group of hypopigmented yellow deposits was observed clinically superonasal to the fovea in the left eye on Day 28 (ellipse) and Day 90 (ellipse and circle) assessments. Note increasing retinal changes on Day 28 compared to day 90 (ellipse) and new area of RPE changes in circle on Day 90. (Images taken at $20^{\circ}$ )

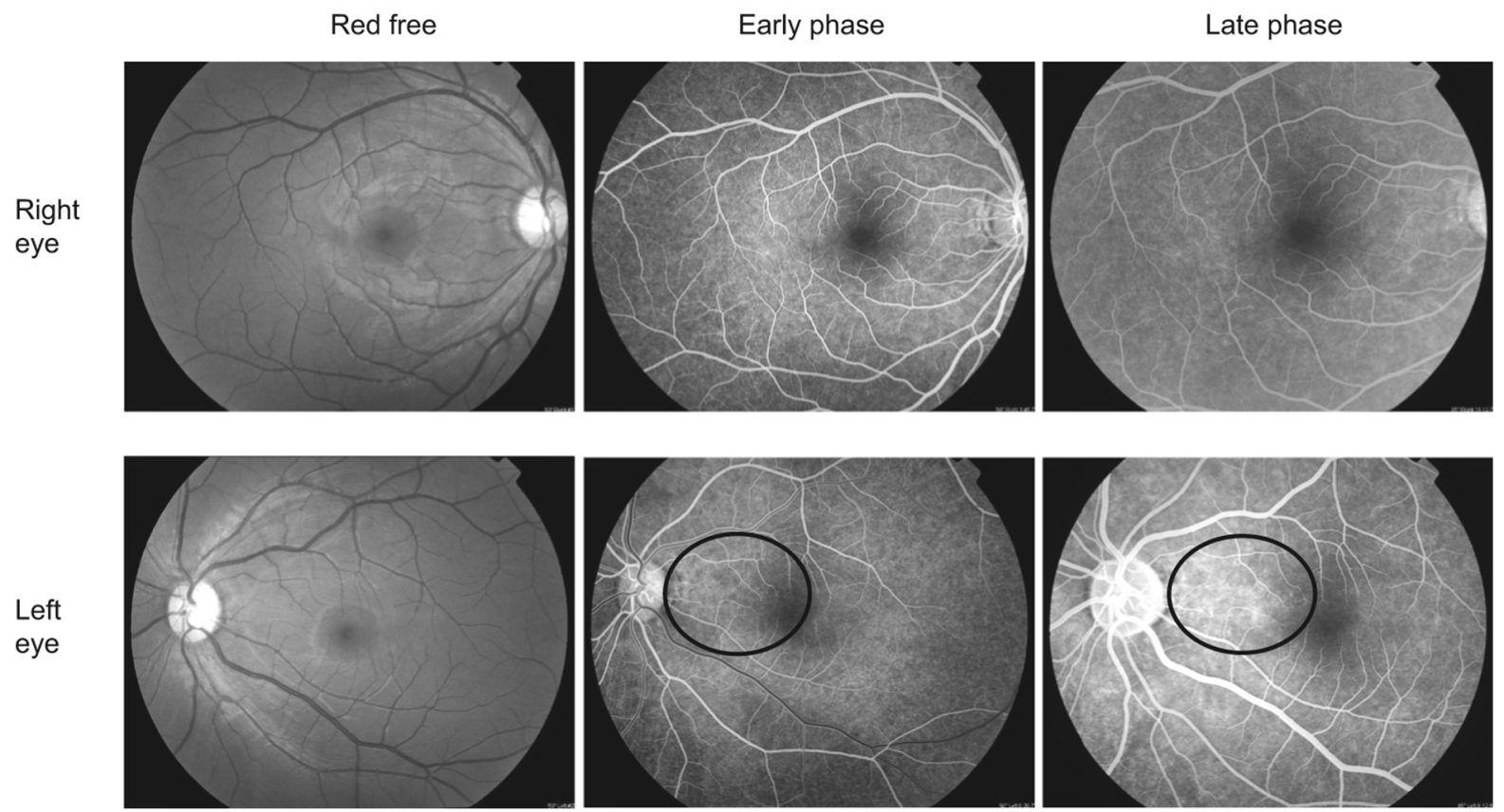

Fig. 5 Fundus fluorescein angiograms performed on Day 28 in the same patient in the tafenoquine group as for Fig. 4, showed retinal pigment epithelium abnormalities in the left eye as pinpoints of hyperfluorescence in the early phase of the angiograms, persisting through the late frames (circled area). These hyperfluorescence spots corresponded to the hypopigmented area mostly involving the papillomacular bundle and supero-nasal to the fovea (circled areas). (Images taken at $35^{\circ}$ ) 
Table 3 Macular function test outcomes in P. vivax malaria patients treated with tafenoquine monotherapy or chloroquine/primaquine sequential therapy at each assessment

\begin{tabular}{|c|c|c|c|c|c|c|c|}
\hline \multirow[t]{2}{*}{ Outcome } & \multirow[t]{2}{*}{ Eye } & \multicolumn{3}{|l|}{ Tafenoquine } & \multicolumn{3}{|c|}{ Chloroquine/primaquine } \\
\hline & & $\begin{array}{l}\text { Baseline } \\
(N=46)\end{array}$ & $\begin{array}{l}\text { Day } 28 \\
(N=44)\end{array}$ & $\begin{array}{l}\text { Day } 90 \\
(N=37)\end{array}$ & $\begin{array}{l}\text { Baseline } \\
(N=24)\end{array}$ & $\begin{array}{l}\text { Day } 28 \\
(N=24)\end{array}$ & $\begin{array}{l}\text { Day } 90 \\
(N=22)\end{array}$ \\
\hline \multirow{4}{*}{$\begin{array}{l}\text { High contrast visual acuity, } \\
\text { LogMAR }\end{array}$} & Left & $0.31(0.018)$ & $0.31(0.013)$ & $0.31(0.010)$ & $0.31(0.014)$ & $0.31(0.011)$ & $0.30(0.010)$ \\
\hline & & {$[0.22-0.34]$} & {$[0.30-0.34]$} & {$[0.30-0.34]$} & {$[0.30-0.34]$} & {$[0.30-0.34]$} & {$[0.30-0.34]$} \\
\hline & Right & $0.31(0.019)$ & $0.31(0.013)$ & $0.31(0.010)$ & $0.31(0.014)$ & $0.31(0.009)$ & $0.31(0.12)$ \\
\hline & & {$[0.22-0.34]$} & {$[0.30-0.34]$} & {$[0.30-0.34]$} & {$[0.30-0.34]$} & {$[0.30-0.32]$} & {$[0.30-0.34]$} \\
\hline \multirow[t]{2}{*}{ Amsler grid, abnormal, $n(\%)$} & Left & 0 & 0 & 0 & 0 & 0 & 0 \\
\hline & Right & 0 & 0 & 0 & 0 & 0 & 0 \\
\hline \multirow{2}{*}{$\begin{array}{l}\text { Humphrey }^{\mathrm{TM}} 10-2 \text {, abnormal, } \\
n(\%)\end{array}$} & Left & 0 & $2(4.5)$ & $4(10.8)$ & 0 & $3(12.5)$ & 0 \\
\hline & Right & $1(2.2)$ & $4(9.1)$ & $1(2.7)$ & 0 & $2(8.3)$ & 0 \\
\hline \multirow{4}{*}{$\begin{array}{l}\text { Macular stress test, recovery, } \\
\text { secs }\end{array}$} & Left & $3.5(1.6)$ & $3.0(1.0)$ & $2.9(1.1)$ & $4.4(3.8)$ & $3.3(1.3)$ & $2.9(0.8)$ \\
\hline & & {$[1-9]$} & {$[2-6]$} & [2-8] & [1-18] & [2-8] & {$[2-4]$} \\
\hline & Right & 3.5 (1.6) & $3.0(1.1)$ & $2.7(0.9)$ & $3.6(1.8)$ & $3.3(1.6)$ & $2.8(0.7)$ \\
\hline & & [2-9] & {$[2-6]$} & {$[1-6]$} & [2-9] & [2-8] & {$[2-4]$} \\
\hline \multirow{4}{*}{$\begin{array}{l}\text { Color perception PIP, plates } \\
\text { missed }\end{array}$} & Left & $0.1(0.4)$ & $0(0.3)$ & 0 & 0 & 0 & 0 \\
\hline & & {$[0-3]$} & {$[0-2]$} & & & & \\
\hline & Right & $0.1(0.4)$ & $0(0.3)$ & 0 & 0 & 0 & 0 \\
\hline & & {$[0-3]$} & {$[0-2]$} & & & & \\
\hline \multirow{4}{*}{$\begin{array}{l}\text { Color perception L'Anthony } \\
\text { hue } 40 \text {, score }\end{array}$} & Left & $2.3(2.9)$ & $1.6(2.0)$ & $1.2(1.5)$ & $2.3(2.4)$ & $0.9(1.2)$ & $1.5(1.7)$ \\
\hline & & [0-12] & {$[0-8]$} & {$[0-4]$} & [0-9] & {$[0-4]$} & {$[0-4]$} \\
\hline & Right & $2.7(3.0)$ & $1.6(2.1)$ & $1.4(1.6)$ & $2.1(3.0)$ & $1.1(1.6)$ & $1.3(1.3)$ \\
\hline & & {$[0-11]$} & {$[0-8]$} & {$[0-6]$} & {$[0-12]$} & {$[0-6]$} & {$[0-4]$} \\
\hline
\end{tabular}

Values are mean (SD) [range] unless otherwise indicated

NB: The patient in the chloroquine/primaquine group with baseline bilateral retinal dot hemorrhage is included in this table of results

and the CQ/PQ group (3/24 [12.5\%]) had an abnormal Humphrey ${ }^{\mathrm{TM}}$ 10-2 test (Tables 2, 3).

In the TQ group at Day 28, 4/44 (9.1\%) patients had an abnormal Humphrey ${ }^{\mathrm{TM}}$ 10-2 test. At Day 90, three of the four cases had resolved with the remaining patient still having an abnormal Humphrey ${ }^{\mathrm{TM}} 10-2$ test in both eyes. However, at Day 90 a further three patients developed an abnormal Humphrey ${ }^{\mathrm{TM}}$ 10-2 test, all in the left eye. In the CQ/PQ group, three patients had abnormal Humphrey ${ }^{\mathrm{TM}}$ 10-2 test results at Day 28, bilateral in two cases and one case in the left eye. Thus, at Day 90, 4/37 (10.8\%) patients in the TQ group versus $0 / 22(0 \%)$ in the CQ/PQ arm had an abnormal Humphrey ${ }^{\mathrm{TM}} 10-2$ test $(P=0.117$; treatment difference $10.8 \%, 95 \%$ CI $-5.11,25.6)$. Of those patients who had abnormal Humphrey 10-2 tests, one in each treatment group had concurrent retinal findings, i.e. pigment changes in both eyes on Day 28 and Day 90 in the TQ group, and in the CQ/PQ group, mild pigment abnormality, grade 1 in both eyes on Day 28. In all cases in both treatment groups, decreases in sensitivity were $<5 \mathrm{~dB}$ and not considered to be clinically relevant.

\section{Discussion}

Retinal hemorrhage related to $P$. vivax

This prospective study of patients infected with $P$. vivax showed that retinal hemorrhage occurred in $2.9 \%(2 / 70)$ of patients. At baseline, one patient had a small retinal hemorrhage in both eyes at the fovea. There was also one retinal hemorrhage at Day 90 in the 
TQ group. Ocular involvement, including retinopathies, is known to occur with $P$. falciparum malaria in adults and children, and the frequency of retinal changes increases with the severity of the disease [22]. In $P$. vivax malaria, retinal hemorrhage has been reported but appears to be a rare occurrence; with only six previously published cases [23, 24]. In Choi's series, hemorrhage can be either pre-retinal/subhyaloid or retinal and can involve one or both eyes with the recovery period of 1 week to 5 months [24].

Ophthalmic data on TQ

This study reported ophthalmic data from a clinical efficacy and safety trial of TQ monotherapy versus $\mathrm{CQ} / \mathrm{PQ}$ sequential therapy in patients with $P$. vivax malaria. It is the first study to show that short-course (3-day) TQ can cause mild keratopathy. The study also collected data to evaluate for possible retinopathy, though results were inconclusive. The $1200 \mathrm{mg}$ total TQ dose was evaluated to see whether higher doses were more effective for radical cure as monotherapy, but also provides a vital safety dataset at four times the single TQ dose (300 mg) used in Phase III trials for $P$. vivax radical cure in combination with CQ $(1500 \mathrm{mg}$ free base). The information from this study thus continues to build the ocular safety database for this important new drug.

In the TQ group, by direct slit-lamp examination, keratopathy was reported in 14/44 (31.8\%) evaluable patients. In these patients, there was no effect on vision and the keratopathy observed was considered to be benign and reversible. Structurally, TQ has cationic amphiphilic characteristics such that an effect on the cornea might be expected. Phospholipidosis is associated with amphiphilic drugs, such as aminoquinoline antimalarials, and can result in accumulation of intracellular phospholipids which cannot be metabolized by lysosomal phospholipases. These deposits are known to occur with CQ in the corneal epithelium and superficial stroma [17]. They may first appear as a Hudson-Stähli line or an increase in a preexisting Hudson-Stähli line. They are more commonly seen as a whorl-like pattern known as cornea verticillata, which can be seen with other amphiphilic drugs, such as amiodarone or chlorpromazine [17]. In this study, keratopathy appeared after only three large TQ doses (total dose $1200 \mathrm{mg}$ ). This may be related to the TQ half-life of about 17.5 days (range 4-36 days) and a large volume of distribution, suggesting extensive tissue binding [25-27]. However, whether there is a direct relationship between TQ pharmacokinetics and keratopathy has yet to be determined.

Because direct examinations were assessed from the cornea to the retina, the presence of keratopathy could have unintentionally unblinded the study. However, that there were more reports of retinal abnormalities described as mild mottling of the retinal pigment epithelium or hypopigmentation in the TQ $(10 / 44,22.7 \%)$ versus CQ/PQ $(2 / 24,8.3 \%)$ group; that 7 TQ patients had both keratopathy and retinopathy versus none in the CQ/PQ group; and that retinal changes remote from macula at Day 28 and Day 90 versus baseline in one TQ patient suggested increasing morphological changes (despite no clinically relevant changes in visual acuity or decreased visual field sensitivity) indicates that possible correlations between keratopathy and retinopathy, and in one case early progressive retinal epithelium changes, related to TQ cannot be ruled out.

Visual field testing (Humphrey ${ }^{\mathrm{TM}}$ 10-2) was the most sensitive objective test of retinal toxicity used in this study $[15,21]$. Post-baseline, a similar frequency of mildly decreased sensitivity was seen; 7/44 (15.9\%) with TQ and 3/24 (12.5\%) with CQ/PQ. In all cases, changes were $<5 \mathrm{~dB}$; below the threshold considered clinically relevant. In all but three cases, there were no concurrent findings on retinal examination. In the $\mathrm{CQ} /$ PQ group, all of the abnormal Humphrey ${ }^{\mathrm{TM}} 10-2$ tests had resolved by Day 90. In the TQ group, four patients had abnormal Humphrey ${ }^{\mathrm{TM}}$ 10-2 tests at Day 90; of these, one patient continued to have a decreased sensitivity from Day 28 (in both eyes) and three patients had abnormal results emerging at this assessment (in one eye only). It is not clear why such findings should appear so late during follow-up in these three patients.

The current study was limited by the recommended methods at the time that it was conducted, whereas more sensitive tests to evaluate drug ocular toxicity are now available. Recent guidelines from the American Academy of Ophthalmology (AAO) recommend Humphrey ${ }^{\mathrm{TM}}$ 10-2 fields for screening after prolonged CQ treatment plus the use of one or more of the following objective tests: multifocal electroretinogram (mfERG), spectral domain optical coherence tomography (SD-OCT), and fundus autofluorescence (FAF) [15]. When Humphrey ${ }^{\mathrm{TM}}$ 10-2 fields are 
performed independently, even the most subtle changes are an indication for objective testing. The study reported in this paper was conducted before this AAO guidance, and is limited in its conclusions as these investigations were not performed [15]. Also, guidelines suggest using a person's height for calculating drug dose, rather than body weight, as this can lead to overmedication with CQ in obese patients $[28,29]$. A study investigating ocular toxicity with TQ versus placebo in healthy volunteers has recently been completed and includes SD-OCT and FAF in addition to standard clinical assessments (ClinicalTrials.gov Identifier: NCT02658435). Conducting these assessments within a malaria treatment clinical trial would be challenging as the necessary facilities would not normally be available at the point of care.

The first study to report ocular safety of TQ was conducted in healthy volunteers receiving TQ $200 \mathrm{mg}$ weekly for 6 months in Bethesda, MD and Chiltern, UK [30], though is not directly comparable to the current study as different macular function tests were used and there were no clinical retinal examinations, only retinal photography. In the 70 subjects receiving TQ available for assessment, there was no effect on night vision (low-contrast visual acuity test, mesopic contrast threshold tests, and scotopic contrast test) compared with 32 subjects receiving placebo [30]. Forward light scatter test and macular functions were also similar between the two groups after 6 months of TQ therapy [30]. Based on digital retinal photographs, retinal abnormalities were noted in one patient in each study arm. In the subject receiving TQ, a single area of retinal hyperpigmentation was detected at follow-up, without any decline in visual acuity, foveal sensitivity, or change in visual field, and remained unchanged for 11 months after therapy cessation [30]. The subject receiving placebo had a retinal abnormality noted at 12 weeks that resolved within 2 months. Independent retinal photograph grading was performed for 39 subjects receiving TQ and 21 in the placebo group with no findings of changes in retinal morphology or any objective signs of toxicity [30]. Humphrey ${ }^{\mathrm{TM}} 10-2$ tests were performed, with no apparent trends in the rate of timing of abnormal results [30]. However, one subject receiving TQ $(n=56)$ was withdrawn at week 3 for an abnormal Humphrey ${ }^{\mathrm{TM}} 10-2$ test; there was no effect on vision and the mild decrease in macular sensitivity resolved spontaneously [30].
A previous study of long-term TQ prophylaxis described ocular effects. In the study, Australian soldiers received TQ $200 \mathrm{mg}$ weekly for 6 months. Whorl-like formations and mild vortex keratopathy were observed in 69/74 (93.2\%) subjects [20]. The changes were reversible and resolved by 1 year and did not adversely affect visual acuity [20]. In these subjects, retinal abnormalities were also noted on clinical examination in 27/69 $(39.1 \%)$ receiving TQ (e.g. granularity/pigmentation of the retinal pigment epithelium or hard drusen) versus 4/17 (23.5\%) receiving mefloquine (MQ). Retinal FFAs were performed on subjects with possible retinal findings; 14 receiving TQ, 1 receiving MQ. Of these, four TQ subjects and one MQ subject had FFAs that were considered possibly abnormal [20]. However, review of these data by an Independent Expert Ophthalmology Review Board concluded that the retinal findings could have been normal variations and that there was no evidence of drug-related visual disturbances. The study was limited in that retinal fundoscopic examinations were unmasked at follow-up because the presence of corneal deposits was already known to investigators. Also, there were no baseline data present for comparison. Humphrey ${ }^{\mathrm{TM}}$ 10-2 results were normal in all subjects $(N=63)$ in the TQ group and in 15/16 (93.8\%) of subjects in the MQ group [20]. These data, taken together with the Bethesda volunteer study described above, provided no conclusive evidence of early retinal changes related to TQ, though the number of subjects examined was small. Note that subjects receiving prophylaxis received much larger TQ doses, and for longer, than for $P$. vivax radical cure.

Compared to the prophylaxis study in Australian soldiers, the shorter TQ dose duration used in the current trial might have led to the decreased frequency and severity of the ocular findings noted. However, our results are consistent with a trial in healthy volunteers in the USA and UK, in which 15/60 (25.0\%) subjects given TQ $200 \mathrm{mg}$ weekly for 6 months had mild keratopathy compared with 4/25 (16.0\%) in the placebo arm [30]. The differences between these studies may reflect differences in data collection methods, data quality, environmental exposure, ethnic or other unknown factors. Sufficient data are not yet available to allow comment on the relationship between TQ dose and duration and the incidence of keratopathy. 
In studies investigating $P$. vivax radical cure, two older reports of the effect of TQ at doses ranging from $500 \mathrm{mg}$ single dose to $2100 \mathrm{mg}$ over 7 days after treatment with CQ $(N=90)$ did not include ophthalmic safety data specifically [8, 9]. However, 4/90 (4.4\%) patients receiving TQ experienced an eyerelated adverse event (one each of conjunctivitis, eye irritation, blurred vision and ocular hyperemia); there were no instances in the comparator groups (CQ and $\mathrm{CQ} / \mathrm{PQ}$ ) [31]. A recent, much larger Phase IIb randomized dose-ranging trial included 329 patients with $P$. vivax malaria who received TQ doses of $50 \mathrm{mg}, 100 \mathrm{mg}, 300 \mathrm{mg}$ or $600 \mathrm{mg}$ as a single-dose plus 3-day CQ (total CQ dose $1500 \mathrm{mg}$ free base equivalent) or $\mathrm{CQ} / \mathrm{PQ}$ or $\mathrm{CQ}$ alone [14]. Adverse events attributed to eye disorders occurred in $5 / 225$ $(2.2 \%)$ of patients that received TQ/CQ (2 conjunctivitis, 2 eye pain, 1 blurred vision, 1 conjunctivial hyperemia) versus $2 / 50(4.0 \%)$ with $\mathrm{CQ} / \mathrm{PQ}$ (both blurred vision) and 2/54 (4.0\%) with CQ alone (both blurred vision) [14]. Ophthalmic assessments were conducted in 93 patients overall, with no reports of keratopathy in any treatment group [14]. There were mild post-baseline transient changes in the results of the Humphrey visual field test in 7/61 (11\%) patients receiving $\mathrm{TQ} / \mathrm{CQ}, 1 / 15(7 \%)$ with $\mathrm{CQ} / \mathrm{PQ}$, and in $1 / 17$ (5.9\%) with CQ alone. In all cases, these findings had resolved by Day 180 of the study. There were no other clinically important ophthalmic findings [14].

In the current study, despite the known ocular toxicity of $\mathrm{CQ}$, keratopathy or clinically relevant ocular changes were not reported in the $\mathrm{CQ} / \mathrm{PQ}$ group. These findings are consistent with the short (3-day) dose duration of CQ in antimalarial therapy. Ocular complications, such as corneal opacity, reversible retinal lipidosis, and irreversible receptor cell degeneration have been documented with $\mathrm{CQ}$, generally during extended use in rheumatoid diseases $[15,17,32,33]$. The risk of ocular complications increases with therapy duration, and CQ doses of $250 \mathrm{mg}$ or below are rarely associated with ocular side effects if used for less than 6 months [15, 17]. A retrospective study of CQ ocular toxicity among 155 Thai patients aged 10-70 years, who received treatment for 6-14 years with a cumulative dose of 26-1771 g, found that early retinal toxicity occurred at 9 months of treatment, with an overall prevalence of keratopathy of $9.0 \%(17 / 155)$ and retinopathy of $14.2 \%$ (22/155), without correlation between keratopathy and retinopathy [32]. Rheumatologists have switched from using CQ to hydroxychloroquine (HCQ) because of its better safety profile in long-term use. The risk of developing HCQ retinopathy is rare and the cumulative dose appeared to be a more important factor than daily dosage [19]. Retinal toxicity associated with CQ and HCQ may continue to progress despite cessation of the drug therapy [19]. The largest study, in 400 Greek patients with rheumatoid arthritis or systemic lupus treated with $<6.5 \mathrm{mg}$ / $\mathrm{kg}$ /day HCQ for a mean of 8.7 years reported the incidence of irreversible retinal toxicity as $0.5 \%$ [34].

\section{Conclusions}

TQ is a promising antimalarial for $P$. vivax radical cure and is the only drug in development as an alternative to PQ for the eradication of $P$. vivax hypnozoites. This study was designed to evaluate TQ efficacy and safety and was not powered to evaluate ophthalmic risk, but provides safety data for TQ concentrations above the expected therapeutic dose. The data suggest that ophthalmic adverse events with TQ $400 \mathrm{mg} /$ day for 3 days tended to be more common than with $\mathrm{CQ} / \mathrm{PQ}$.

There was no evidence of clinically relevant ocular toxicity with either treatment. This is the first study to show mild keratopathy with short-course TQ (total dose $1200 \mathrm{mg}$ ), with no conclusive evidence of early retinal changes, though possible increasing early retinal pigment epithelial changes related to TQ cannot be ruled out in one patient. Although there were some abnormal Humphrey ${ }^{\mathrm{TM}}$ 10-2 visual field tests in patients receiving TQ, sensitivity was decreased by $<5 \mathrm{~dB}$ and vision was not affected. Ophthalmic monitoring will continue in the current TQ Phase III program for $P$. vivax radical cure using the lower TQ $300 \mathrm{mg}$ single-dose plus 3-day CQ regimen (1500 $\mathrm{mg}$ total dose free base; NCT01376167), or TQ $300 \mathrm{mg}$ plus or dihydroartemisinin-piperaquine (320:40 mg; Clinicaltrials.gov: NCT02802501).

Acknowledgements The authors thank the following local study coordinators: Nillawan Buathong, Krisda Jongskul, and Punnee Pitisutithum. The authors recognize the contribution of the following co-investigators: Harald Noedl, MD (Institute of Specific Prophylaxis and Tropical Medicine, Medical University Vienna, Austria); Sombat Teeprasertsuk, MD (Faculty of Tropical Medicine, Mahidol University, Bangkok, 
Thailand), Udomsak Silachamroon, MD (Faculty of Tropical Medicine, Mahidol University, Bangkok, Thailand); Weerapong Phumratanaprapin, MD (Faculty of Tropical Medicine, Mahidol University, Bangkok, Thailand). We thank the Ophthalmology Advisory Board for their contribution: Michael Altaweel, MD (University of Wisconsin, Madison, WI, USA); Douglas A Jabs, MD, MBA (The Johns Hopkins University Bloomberg School of Public Health, Baltimore, MD, USA); Tony Moore, FRCS, FRCOphth, FMedSci (University College London, United Kingdom); Sukhuma Warrasak, MD (Ramathibodi Faculty Hospital, Mahidol University, Bangkok, Thailand). We thank Justin Green for critical comments on the manuscript. Editorial support in the form of editorial suggestions to draft versions of this paper, collecting author comments, copyediting, and graphic services were provided by Naomi Richardson at Magenta Communications Ltd and was funded by GlaxoSmithKline; administrative support was provided by Alex Lowe of Fishawack and was funded by GlaxoSmithKline.

Authors' contributions SW, AE, RSM, MMF, and CO contributed to the study design. SW and AE conducted the ophthalmology examinations, drafted the manuscript, and provided technical support and expertise. SK and MMF supervised the entire study and assisted with study design and data interpretation. MI was involved in acquisition of data, data analysis, and interpretation. CO, MMF, and RSM conceived of and designed this study and wrote the grant which funded it. $\mathrm{CO}$ led the effort to address the safety signals identified in a malaria prophylaxis trial (Nasveld et al. Antimicrob Agents Chemother 2010; 54:792-798 Ref [18 in manuscript]), provided study supervision throughout the study, and edited this manuscript. All authors were involved in the interpretation of the data, contributed to this paper and approved the final version for publication. Sornchai Looareesuwan, MD, who died in July 2007, contributed to this study in the development of the protocol and collection of data.

Funding This publication was made possible by Grant Numbers RC1AI048874-01 and UC1AI049499-01 from the National Institute of Allergy and Infectious Diseases. This study was supported by US Army Medical Research and Material Command and GlaxoSmithKline (Brentford, Middlesex, UK). The US Army Medical Research and Material Command and GlaxoSmithKline were involved in study design, collection and interpretation of data and preparation of this paper.

\section{Compliance with ethical standards}

Conflict of interest The authors have no proprietary or commercial interest in any materials discussed in this article.

Ethics approval and consent to participate This study was conducted in accordance with Good Clinical Practice and the guiding principles of the Declaration of Helsinki. The protocol was approved by the Ethical Committee, Faculty of Tropical Medicine, Mahidol University, Bangkok, Thailand, the Ministry of Public Health, Nonthaburi, Thailand, and the Human Subjects Research Review Board, U.S. Army Medical Research and Material Command, Office of Regulatory Compliance and
Quality, Fort Detrick, MD, USA. All participants provided written informed consent before entry into the study. The protocol can be obtained from the corresponding authors. The trial is registered at Clinicaltrials.gov identifier: NCT01290601.

Open Access This article is distributed under the terms of the Creative Commons Attribution 4.0 International License (http:// creativecommons.org/licenses/by/4.0/), which permits unrestricted use, distribution, and reproduction in any medium, provided you give appropriate credit to the original author(s) and the source, provide a link to the Creative Commons license, and indicate if changes were made.

\section{References}

1. Guerra CA, Howes RE, Patil AP, Gething PW, Van Boeckel TP, Temperley WH, Kabaria CW, Tatem AJ, Manh BH, Elyazar IR, Baird JK, Snow RW, Hay SI (2010) The international limits and population at risk of Plasmodium vivax transmission in 2009. PLoS Negl Trop Dis 4:e774. https://doi.org/10.1371/journal.pntd.0000774

2. Howes RE, Battle KE, Mendis KN, Smith DL, Cibulskis RE, Baird JK, Hay SI (2016) Global epidemiology of Plasmodium vivax. Am J Trop Med Hyg 95:15-34. https:// doi.org/10.4269/ajtmh.16-0141

3. Mendis K, Sina BJ, Marchesini P, Carter R (2001) The neglected burden of Plasmodium vivax malaria. Am J Trop Med Hyg 64:97-106

4. Price RN, Douglas NM, Anstey NM (2009) New developments in Plasmodium vivax malaria: severe disease and the rise of chloroquine resistance. Curr Opin Infect Dis 22:430-435. https://doi.org/10.1097/QCO.0b013e32832f14c1

5. Galappaththy GN, Tharyan P, Kirubakaran R (2013) Primaquine for preventing relapse in people with Plasmodium vivax malaria treated with chloroquine. The Cochrane database of systematic reviews CD004389. https://doi.org/ 10.1002/14651858.cd004389.pub3

6. Ponsa N, Sattabongkot J, Kittayapong P, Eikarat N, Coleman RE (2003) Transmission-blocking activity of tafenoquine (WR-238605) and artelinic acid against naturally circulating strains of Plasmodium vivax in Thailand. Am J Trop Med Hyg 69:542-547

7. Coleman RE (1990) Sporontocidal activity of the antimalarial WR-238605 against Plasmodium berghei ANKA in Anopheles stephensi. Am J Trop Med Hyg 42:196-205

8. Walsh DS, Looareesuwan S, Wilairatana P, Heppner DG Jr, Tang DB, Brewer TG, Chokejindachai W, Viriyavejakul P, Kyle DE, Milhous WK, Schuster BG, Horton J, Braitman DJ, Brueckner RP (1999) Randomized dose-ranging study of the safety and efficacy of WR 238605 (Tafenoquine) in the prevention of relapse of Plasmodium vivax malaria in Thailand. J Infect Dis 180:1282-1287. https://doi.org/10. 1086/315034

9. Walsh DS, Wilairatana P, Tang DB, Heppner DG Jr, Brewer TG, Krudsood S, Silachamroon U, Phumratanaprapin W, Siriyanonda D, Looareesuwan S (2004) Randomized trial of 3-dose regimens of tafenoquine (WR238605) versus lowdose primaquine for preventing Plasmodium vivax malaria 
relapse. Clin Infect Dis 39:1095-1103. https://doi.org/10. $1086 / 424508$

10. Shanks GD, Oloo AJ, Aleman GM, Ohrt C, Klotz FW, Braitman D, Horton J, Brueckner R (2001) A new primaquine analogue, tafenoquine (WR 238605), for prophylaxis against Plasmodium falciparum malaria. Clin Infect Dis 33:1968-1974. https://doi.org/10.1086/324081

11. Dow GS, Gettayacamin M, Hansukjariya P, Imerbsin R, Komcharoen S, Sattabongkot J, Kyle D, Milhous W, Cozens S, Kenworthy D, Miller A, Veazey J, Ohrt C (2011) Radical curative efficacy of tafenoquine combination regimens in Plasmodium cynomolgi-infected Rhesus monkeys (Macaca mulatta). Malaria J 10:212. https://doi.org/10.1186/14752875-10-212

12. Brueckner RP, Lasseter KC, Lin ET, Schuster BG (1998) First-time-in-humans safety and pharmacokinetics of WR 238605, a new antimalarial. Am J Trop Med Hyg 58:645-649

13. Fukuda MM, Krudsood S, Mohamed K, Green JA, Warrasak S, Noedl H, Euswas A, Ittiverakul M, Buathong N, Sriwichai S, Miller RS, Ohrt C (2017) A randomized, double-blind, active-control trial to evaluate the efficacy and safety of a three day course of tafenoquine monotherapy for the treatment of Plasmodium vivax malaria. PLoS ONE 12:e0187376. https:// doi.org/10.1371/journal.pone.0187376

14. Llanos-Cuentas A, Lacerda MV, Rueangweerayut R, Krudsood S, Gupta SK, Kochar SK, Arthur P, Chuenchom N, Mohrle JJ, Duparc S, Ugwuegbulam C, Kleim JP, Carter N, Green JA, Kellam L (2014) Tafenoquine plus chloroquine for the treatment and relapse prevention of Plasmodium vivax malaria (DETECTIVE): a multicentre, double-blind, randomised, phase $2 \mathrm{~b}$ dose-selection study. Lancet 383:1049-1058. https://doi.org/10.1016/S01406736(13)62568-4

15. Marmor MF, Kellner U, Lai TY, Melles RB, Mieler WF, American Academy of Ophthalmology (2016) Recommendations on screening for chloroquine and hydroxychloroquine retinopathy (2016 revision). Ophthalmology 123:1386-1394. https://doi.org/10.1016/j.ophtha.2016.01.058

16. Izazola-Conde C, Zamora-de la Cruz D, Tenorio-Guajardo G (2011) Ocular and systemic adverse effects of ophthalmic and non ophthalmic medications. Proc West Pharmacol Soc 54:69-72

17. Prokopich C, Bartlett J, Jaanus S (2007) Ocular adverse drug reactions to systemic medications. In: Bartlett J, Jaanus $\mathrm{S}$ (eds) Clinical ocular pharmacology. Butterworth-Heinemann, Oxford, pp 701-759

18. Mecklenburg L, Schraermeyer U (2007) An overview on the toxic morphological changes in the retinal pigment epithelium after systemic compound administration. Toxicol Pathol 35:252-267. https://doi.org/10.1080/01926230601178199

19. Michaelides M, Stover NB, Francis PJ, Weleber RG (2011) Retinal toxicity associated with hydroxychloroquine and chloroquine: risk factors, screening, and progression despite cessation of therapy. Arch Ophthalmol 129:30-39. https:// doi.org/10.1001/archophthalmol.2010.321

20. Nasveld PE, Edstein MD, Reid M, Brennan L, Harris IE, Kitchener SJ, Leggat PA, Pickford P, Kerr C, Ohrt C, Prescott W, Tafenoquine Study Team (2010) Randomized, double-blind study of the safety, tolerability, and efficacy of tafenoquine versus mefloquine for malaria prophylaxis in nonimmune subjects. Antimicrob Agents Chemother 54:792-798

21. Dersu I, Wiggins M (2006) Understanding visual fields, part II; Humphrey visual fields. J Ophthalmic Med Technol. http://citeseerx.ist.psu.edu/viewdoc/download;jsessionid= 3DDE9F18027042E8D67188FED3AA583A?doi=10.1.1. 567.1948\&rep=rep1\&type=pdf. Accessed 2 April 2018

22. Abu Sayeed A, Maude RJ, Hasan MU, Mohammed N, Hoque MG, Dondorp AM, Faiz MA (2011) Malarial retinopathy in Bangladeshi adults. Am J Trop Med Hyg 84:141-147. https://doi.org/10.4269/ajtmh.2011.10-0205

23. Lee JH, Chin HS, Chung MH, Moon YS (2010) Retinal hemorrhage in Plasmodium vivax malaria. Am J Trop Med Hyg 82:219-222. https://doi.org/10.4269/ajtmh.2010.090439

24. Choi HJ, Lee SY, Yang H, Bang JK (2004) Retinal haemorrhage in vivax malaria. Trans $\mathrm{R}$ Soc Trop Med Hyg 98:387-389. https://doi.org/10.1016/j.trstmh.2003.12.002

25. Charles BG, Miller AK, Nasveld PE, Reid MG, Harris IE, Edstein MD (2007) Population pharmacokinetics of tafenoquine during malaria prophylaxis in healthy subjects. Antimicrob Agents Chemother 51:2709-2715. https://doi. org/10.1128/AAC.01183-06

26. Edstein MD, Kocisko DA, Brewer TG, Walsh DS, Eamsila C, Charles BG (2001) Population pharmacokinetics of the new antimalarial agent tafenoquine in Thai soldiers. Br J Clin Pharmacol 52:663-670

27. Edstein MD, Kocisko DA, Walsh DS, Eamsila C, Charles BG, Rieckmann KH (2003) Plasma concentrations of tafenoquine, a new long-acting antimalarial agent, in thai soldiers receiving monthly prophylaxis. Clin Infect Dis 37:1654-1658. https://doi.org/10.1086/379718

28. Karmel M (2011) Rx side effects: New Plaquenil guidelines and more. American Academy of Ophthalmology. https:// www.aao.org/eyenet/article/rx-side-effects-new-plaquenilguidelines-more. Accessed 2 Feb 2017

29. Marmor MF, Kellner U, Lai TY, Lyons JS, Mieler WF (2011) Revised recommendations on screening for chloroquine and hydroxychloroquine retinopathy. Ophthalmology 118:415-422. https://doi.org/10.1016/j.ophtha.2010.11.017

30. Leary KJ, Riel MA, Roy MJ, Cantilena LR, Bi D, Brater DC, van de Pol C, Pruett K, Kerr C, Veazey JM Jr, Beboso R, Ohrt C (2009) A randomized, double-blind, safety and tolerability study to assess the ophthalmic and renal effects of tafenoquine $200 \mathrm{mg}$ weekly versus placebo for 6 months in healthy volunteers. Am J Trop Med Hyg 81:356-362

31. Hackett D, Harrell E, Miller AK, Kleim J-P, Mohamed K, Nash L, Ohrt C, Rana R. A dose ranging study for the safety and efficacy of W238605/SB252263 in the prevention of relapse of Plasmodium vivax infection in Thailand. Document number: RM2007/00309/00; study SB-252263/047: GlaxoSmithKline, data on file; 2010

32. Puavilai S, Kunavisarut S, Vatanasuk M, Timpatanapong P, Sriwong ST, Janwitayanujit S, Nantiruj K, Totemchokchyakarn K, Ruangkanchanasetr S (1999) Ocular toxicity of chloroquine among Thai patients. Int J Dermatol 38:934-937

33. Araiza-Casillas R, Cardenas F, Morales Y, Cardiel MH (2004) Factors associated with chloroquine-induced retinopathy in rheumatic diseases. Lupus 13:119-124. https://doi.org/10.1191/0961203304lu514oa 
34. Mavrikakis I, Sfikakis PP, Mavrikakis E, Rougas K, Nikolaou A, Kostopoulos C, Mavrikakis M (2003) The incidence of irreversible retinal toxicity in patients treated with hydroxychloroquine: a reappraisal. Ophthalmology 110:1321-1326. https://doi.org/10.1016/S0161-6420(03)00 409-3 